\title{
Names of referees who worked on papers submitted in 2016
}

The Editorial Board gratefully acknowledges the following individuals for their commitment in reviewing the papers submitted to Radioprotection. Their work and the relevance of their analysis allow us to maintain the high quality of our journal.

\author{
AUBINEAU-LANIECE Isabelle, FRANCE \\ AUPIAIS Jean, FRANCE \\ BERARD Philippe, FRANCE \\ BERTHO Jean-Marc, FRANCE \\ BEZ Jérémy, FRANCE \\ BRUN Stéphane, FRANCE \\ CALVEZ Marianne, FRANCE \\ CANTARINHA Alfredo, FRANCE \\ CESSAC Bruno, FRANCE \\ CHAMPION Didier, FRANCE \\ CHAUMET-RIFFAUD Philippe, FRANCE \\ CORTINA Eduardo, BELGIUM \\ DABIN Jérémie, BELGIUM \\ DAVESNE Estelle, FRANCE \\ DERREUMAUX Sylvie, FRANCE \\ DIDIER Damien, France \\ DREUIL Serge, FRANCE \\ DUBLINEAU Isabelle, FRANCE \\ EN NOURHI Karim, FRANCE \\ ENDO Akira, JAPAN \\ FARAH Jad, FRANCE \\ FLAMANT Stéphane, FRANCE \\ FLESCH Klaus, GERMANY \\ FORBES Aurélie, FRANCE \\ FRANCOIS Sabine, FRANCE \\ ONIN Michèle, FRANCE \\ GREMY Olivier, FRANCE
}

\author{
GRESSIER Vincent, FRANCE \\ GRIFFITHS Nina, FRANCE \\ GRISON Stéphane, FRANCE \\ GSCHWIND Régine, FRANCE \\ GUETAT Philippe, FRANCE \\ GUIPAUD Olivier, FRANCE \\ LECOMTE Jean François, FRANCE \\ LOPEZ Maria Antonia, SPAIN \\ MALCHAIR Françoise, BELGIUM \\ MASSON Olivier, FRANCE \\ MERCAT Catherine, FRANCE \\ METIVIER Henri, FRANCE \\ MURITH Christophe, SWITZERLAND \\ NAGAMANI SuriyaMurthy, AUSTRIA \\ PAQUET François, FRANCE \\ PORT Matthias, GERMANY \\ REBIERE François, FRANCE \\ RENAUD Philippe, FRANCE \\ SAIDOU Ous, CAMEROON \\ SARRAZIN Thierry, FRANCE \\ SOUQUES Martine, FRANCE \\ STROUDE Raphaël, SWITZERLAND \\ STRUELENS Lara, BELGIUM \\ TAMPONNET Christian, FRANCE \\ THIERRY CHEF Isabelle, FRANCE \\ THOMASSIN Alain, FRANCE \\ VAILLANT Ludovic, FRANCE
}

